Results Four key deficiencies were identified: 1.The degree to which sexual risk behaviours such as multiple partnerships and inconsistent contraception use predict adverse outcomes remains under-investigated; 2.Surveys frequently use composite measures with complex scoring systems; 3 . Analyses often fail to report on non-response to individual items and the prevalence of exposures; 4. Sexual risk behaviours and morbidity are often reported without measures of absolute risk.

Conclusion There is a strategic need to exploit survey research better in order to embed investigation of risk factors in clinical risk prediction tools. Survey analyses should investigate which sexual risk behaviours warrant different sexual health interventions, and use measures of absolute risk. Researchers should favour questionnaire items that are concise and easy to score, and should report on item non-response and prevalence of exposures. These steps would facilitate the development of brief, acceptable and adequately predictive tools.

\section{P3.163 DIFFERENCES BETWEEN WOMEN ATTENDING SPECIALIST SEXUAL HEALTH CLINICS AND THOSE ATTENDING GENERAL PRACTICES: IMPLICATIONS FOR TARGETING STI TESTING}

${ }^{1}$ Natalie Edelman, ${ }^{2} \mathrm{CM}$ Mercer, ${ }^{1} \mathrm{~J}$ Cassell, ${ }^{3} \mathrm{RO}$ De Visser. ${ }^{1}$ Brighton and Sussex Medical School, Brighton, UK; ${ }^{2}$ University College London, London, UK; ${ }^{3}$ University of Sussex, Brighton, UK

\subsection{6/sextrans-2017-053264.398}

Introduction In Britain sexual health interventions are increasingly provided in community settings such as General Practices (GPs), while Genito-Urinary Medicine clinics have largely been replaced by Sexual Health and for Contraception clinics (SHACs). This study compared women attending GPs with those attending SHAC in an urban setting to investigate how much these populations differ and to assess the extent of sexual risk behaviour in GP populations.

Methods A convenience sample of women aged 16-44 years attending GP and SHAC services in the city of Brighton and Hove was invited to complete a short questionnaire about socio-demographic, psychosocial and sexual behavioural factors.

Results 1288 participated from GPs and 552 women from SHAC. $11.2 \%$ of GP participants and $3.4 \%$ of SHAC participants $(\mathrm{p}<0.0001)$ reported no male sexual partners in the last year. GP participants were less likely than SHAC participants to report 2 or more male sexual partners in the last year (2PP) $(21.5 \%$ versus $60.9 \% \mathrm{p}<0.0001)$ and to report non-use of condoms with two partners in the last year $6.9 \%$ versus $24.2 \% \mathrm{p}<0.0001$. These associations remained after adjusting for the smaller proportion of women $\leq 24$ years among GP participants compared to SHAC $(21.7 \%$ v. $44.5 \%$ p <0.0001). Binge drinking alcohol in the last week was associated with 2PP $(\mathrm{p}<0.0001)$ and was more common among SHAC participants $\leq 24$ years than GP participants $\leq 24$ years $(49.8 \%$ versus $37.9 \% \mathrm{p}=0.009)$. However, it did not differ across sites for women aged $25-44$ years $(33.3 \%$ versus $36.3 \% \mathrm{p}=0.362)$.

Conclusion Although women attending GPs are less likely than those attending SHAC to report sexual risk behaviour in the last year, a substantial minority may benefit from sexual health promotion, STI testing and alcohol awareness. This supports the development of a risk prediction tool to target sexual health and adjunct interventions in community settings among women aged 16-44 years.
P3.164 ANTIBIOTIC RESISTANCE AND MOLECULAR TYPING OF NEISSERIA GONORRHOEAE ISOLATED FROM THE THREE OVERSEAS SITES THROUGH THE GLOBAL EMERGING INFECTIONS SURVEILLANCE AND RESPONSE SYSTEM (GEIS)

${ }^{1}$ Nazia Rahman, ${ }^{2}$ Nicole Kluz, ${ }^{3}$ Naiki Puplampu-Attram, ${ }^{3}$ Andrew Letizia, ${ }^{4}$ Silvia Montano, ${ }^{4}$ James Regeimbal, ${ }^{4}$ Andrea Mccoy, ${ }^{5}$ Tamar Akhvlediani, ${ }^{5}$ Michael Washington, ${ }^{6}$ Olusegun Soge, 'Grace Macalino, ${ }^{1}$ Ann Jerse. 'Uniformed Services University, Bethesda, Md, USA; ${ }^{2}$ University of Texas, Austin, TX, USA; ${ }^{3}$ U.S. Naval Medical Research Unit Number 3. Accra, Ghana; ${ }^{4}$ U.S. Naval Medical Research Unit Number 6, Lima - Peru; ${ }^{5}$ U.S. Army Medical Research Unit, Georgia, Tbilisi, Georgia; ${ }^{6}$ University of Washington, Seattle, USA

\subsection{6/sextrans-2017-053264.399}

Introduction Increasing antibiotic resistance in Neisseria gonorrhoeae (GC) threatens treatment and control measures for gonorrhoea and can affect military readiness. The Global Emerging Infectious Surveillance and Response System of the US Armed Forces Health Surveillance Branch supports a repository for GC isolated at US military treatment facilities in the continental US (CONUS) and at several overseas (OCONUS) labs. Here we report the antibiotic susceptibility and N. gonorrhoeae multi-antigen sequence types (NG-MAST) of isolates collected from three OCONUS sites: Republic of Georgia, Peru and Ghana in 2012-2016.

Methods GC was identified using standard biochemical and serological methods. Susceptibility to ceftriaxone, cefixime (Cfx), azithromycin (Az), gentamicin, penicillin (Pen), tetracycline (Tet), ciprofloxacin (Cip), and spectinomycin was determined by Etest. $\beta$-lactamase ( $(\mathrm{L})$ activity was determined by nitrocefin hydrolysis. NG-MAST was performed using standard methods.

Results Fifty-seven confirmed GC isolates were obtained from the three OCONUS sites. Cip resistance occurred in $74 \%$ (Ghana) and 89\% (Peru) of isolates, with 63.2\% (Ghana) and $28.6 \%$ (Peru) of isolates $\mathrm{Cip}^{\mathrm{R}}$, $\mathrm{Pen}^{\mathrm{R}}$ and $\mathrm{Tet}^{\mathrm{R}}$. Two isolates with reduced susceptibility to $\mathrm{Cfx}$ were identified among isolates from Ghana and Georgia and 18 isolates with reduced susceptibility to $\mathrm{Az}$ were identified across the 3 sites. Over $65 \%$ of isolates from Ghana and Peru produced $3 \mathrm{~L}$. Cip ${ }^{\mathrm{R}}$ strains primarily encoded S91,D95A or S91F,D95G substitutions in GyrA, combined with S87R (Peru) or S87N (Ghana) substitutions in ParC. Interestingly, a high proportion of isolates from Ghana (36.8\%) and Peru $(85.7 \%)$ were of unique NG-MAST types.

Conclusion Multidrug resistant $\mathrm{GC}$ and $\mathrm{BL}$ production are common in these OCONUS sites. Several previously undescribed NG-MAST sequence types were identified in Peru and Ghana, suggesting the GC strains circulating in these countries are different from those in North America, Australia, and Europe. Further surveillance is needed to inform treatment recommendations in OCONUS sites.

\section{P3.165 QUALITY OF LIFE IN WOMEN WITH GENITAL TRACT INFECTIONS BY HUMAN PAPILLOMAVIRUS (HPV)}

${ }^{1}$ Nelson Vespa Junior, ${ }^{1}$ Adriana Bittencourt Campaner, ${ }^{2}$ Mauro Romero Leal Passos, ${ }^{3}$ Paulo Cesar Giraldo. ${ }^{1}$ FCMSCSP, Sao Paulo - SP, Brazil; ${ }^{2}$ UFF, Rio De Janeiro - RJ, Brazil; ${ }^{3}$ UNICAMP, Campinas - SP, Brazil

10.1136/sextrans-2017-053264.400

Introduction Human papillomavirus is sexually transmitted diseases (STDs) are among the most common public health issues 